\title{
Chapter 1. Epidemiology of hypertension
}

\author{
Hypertension Research (2014) 37, 260-265; doi:10.1038/hr.2014.4
}

\section{POINT 1}

1. The number of hypertensive patients in Japan is estimated to be $\sim \mathbf{4 3}$ million (Evidence level: E-III).

2. Cardiovascular disease/stroke/myocardial infarction/chronic kidney disease morbidity and mortality risks increase with blood pressure elevation beyond optimal blood pressure levels (systolic blood pressure: $<120 \mathrm{~mm} \mathrm{Hg}$; and diastolic blood pressure: $<80 \mathrm{~mm} \mathrm{Hg}$; Evidence level: E-la).

3. The annual number of deaths due to hypertension in Japan is estimated to be $\sim 100000$. It follows that of deaths due to smoking. Approximately $50 \%$ of deaths from cardiovascular diseases and $50 \%$ or more of deaths from stroke are estimated to be attributed to high blood pressure levels beyond optimal values (Evidence level: E-la).

4. Among blood pressure parameters, systolic blood pressure more strongly predicts the cardiovascular disease risk. In the presence of other risk factors, this risk increases further (Evidence level: E-la).

5. The mean salt intake among the Japanese remains high. Reducing salt intake is important for lowering the blood pressure levels of the Japanese. Furthermore, the prevalence of obesity-related hypertension has increased (Evidence level: E-III).

6. In Health Japan 21 (II), a 4-mm Hg decrease in the average systolic blood pressure level of the Japanese within 10 years is targeted by promoting strategies for diet/physical activities/ alcohol consumption. If this is achieved, the annual number of deaths from stroke will decrease by $\sim 10,000$ and that of deaths from coronary heart disease will decrease by $\sim 5,000$ (Evidence level: E-la).

\section{CURRENT STATUS OF BLOOD PRESSURE LEVELS IN THE JAPANESE AND THEIR CHANGES}

According to the National Health and Nutrition Survey in 2010, 60 and $45 \%$ of Japanese men and women, respectively, aged over 30 years were regarded as having hypertension (systolic blood pressure: $140 \mathrm{mmHg}$ or more; or diastolic blood pressure: $90 \mathrm{~mm} \mathrm{Hg}$ or more; or treatment with antihypertensive drugs). ${ }^{1}$ An analysis of changes in the hypertension prevalence/treatment/control rates over 30 years, from NIPPON DATA80 (1980) until NIPPON DATA2010 (2010), involving the National Survey of Circulatory Disorders, showed that the prevalence of hypertension was higher at a more advanced age, and that it exceeded $60 \%$ in men aged 50 years or older and in women aged 60 years or older (Figure 1-1a).,3 On the basis of the prevalence of hypertension in NIPPON DATA2010, the number of hypertensive patients in 2010 in Japan was estimated to be $\sim 43$ million (23 million men and 20 million women; Figure 1-2).,3 Although there is a decrease in the prevalence of hypertension in women in each age group, men aged over 50 years may show a plateau or increase. With the rapid aging of society, the number of hypertensive patients in Japan may further increase in the future.

The treatment rate (proportion of patients receiving antihypertensive drugs among those with hypertension) has increased during the past 30 years. The rates were $50 \%$ or more in men and women aged $60-69$ years and $60 \%$ or more in those aged $70-79$ years (Figure 1-1b)., In addition, the control rate (proportion of patients with a blood pressure of $<140 / 90 \mathrm{~mm} \mathrm{Hg}$ among those receiving antihypertensive drugs) has also increased during the past 30 years, reaching $\sim 30$ and $40 \%$ in men and women, respectively (Figure 1-1c). ${ }^{2,3}$

On the other hand, the average systolic blood pressure of the Japanese was higher at a more advanced age regardless of gender. However, in all age groups, it has markedly decreased during the past 50 years (Figure $1-3 \mathrm{a}$ ) ${ }^{3}$ there was a 10 - to $20-\mathrm{mm} \mathrm{Hg}$ decrease in each age group among both men and women. The age-adjusted mortality rate due to stroke in Japan reached a peak in the 1960s. It then rapidly decreased (men 361, women 244, in 1965; men 47, women 26, in 2011 (per 100000 persons)), ${ }^{4}$ and the life expectancy of the Japanese became the longest in the world, which may be largely due to the decrease in average blood pressure among the Japanese. A similar tendency was also noted in other epidemiological studies in Japan., The widespread introduction of hypertension screening in health checkups, advances in hypertension treatment with antihypertensive drugs or its widespread application, and changes in Japanese lifestyle/ environmental factors, such as a decrease in salt intake, may have contributed to such a reduction in the average blood pressure of the Japanese. However, it should be noted that there has been no marked decrease in the average diastolic blood pressure of men aged 30-59 years during the past 50 years (Figure $1-3 \mathrm{~b}$ ). ${ }^{3}$

\section{ASSOCIATION BETWEEN HYPERTENSION AND VARIOUS DISEASES}

1) Hypertension-related increase in the risk for stroke/heart disease Hypertension is the most important risk factor for cardiovascular diseases (stroke and heart disease). In the 1960s, Japan was one of the countries with the highest mortality rate due to stroke. The mortality/ morbidity rates due to stroke are still higher than those due to acute myocardial infarction. ${ }^{7}$ However, the mortality rate due to stroke has markedly decreased during the past 50 years, and the mortality rate from all heart diseases, including heart failure, has become higher than that due to stroke. The age-adjusted mortality rate due to stroke in Japan was about three times higher than that due to acute myocardial infarction. ${ }^{7}$ Several epidemiological studies have also reported that 
a

Prevalence of hypertension*2

\section{Men}

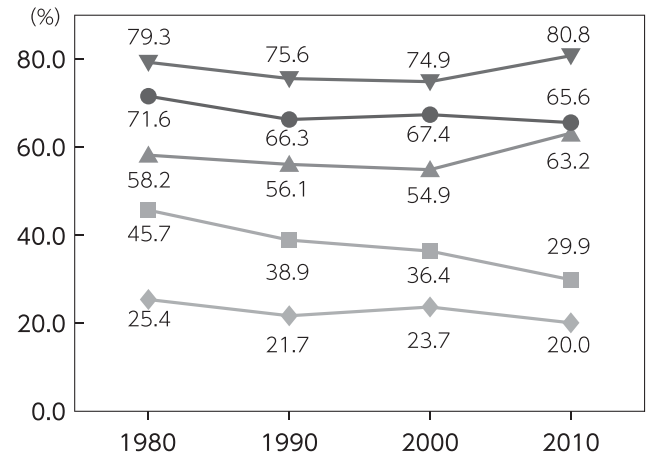

b

Treatment rate*3

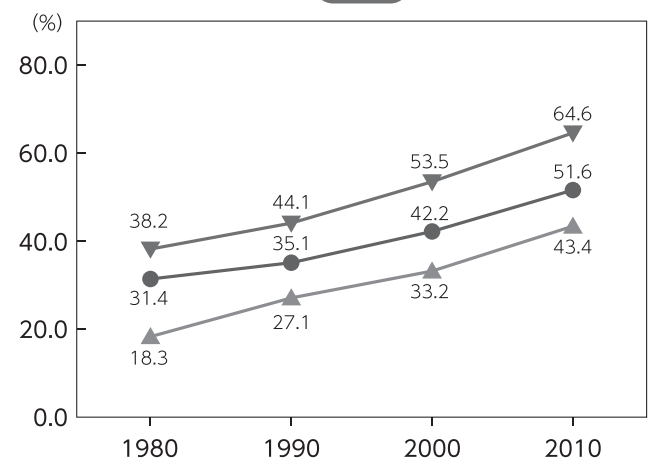

C
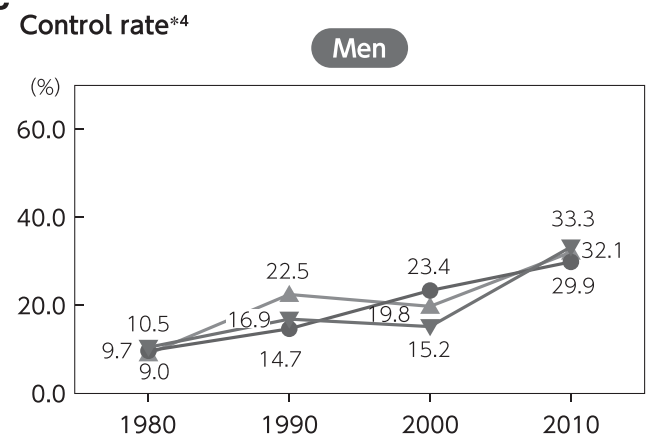

Nomen



Women

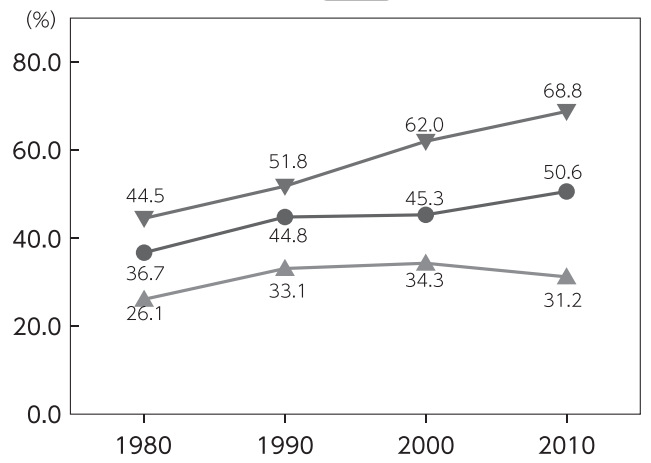

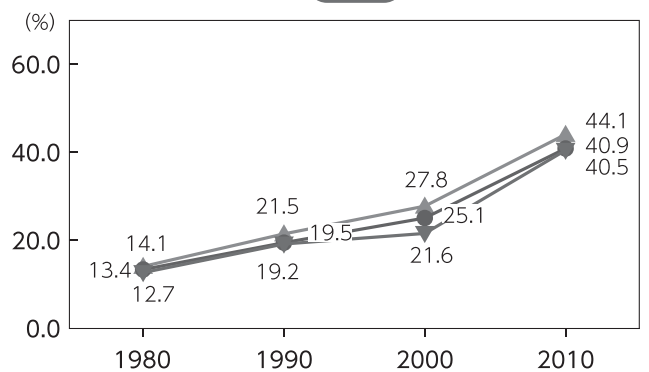

Figure 1-1 The 30-year trend in the prevalence, treatment and control rates of hypertension by sex and age group (1980-2010). 3rd National Survey of Circulatory Disorders (NIPPON DATA80), 4th National Survey of Circulatory Disorders (NIPPON DATA90), 5th National Survey of Circulatory Disorders, NIPPON DATA2010*1). ${ }^{*}$ The 6th National Survey of Circulatory Disorder was not conducted. As a scientific study by the Ministry of Health, Labour and Welfare (designated study), NIPPON DATA2010 was performed. *2Systolic blood pressure of $140 \mathrm{~mm} \mathrm{Hg}$ or more, diastolic blood pressure of $90 \mathrm{~mm} \mathrm{Hg}$ or more and/or treatment with antihypertensive drugs (the first value of two measurements was used in $2000 / 2010$ ). ${ }^{*}$ Proportion of individuals taking antihypertensive drugs among hypertensives. ${ }^{*}$ Proportion of individuals with a systolic blood pressure of $<140 \mathrm{~mm} \mathrm{Hg}$ and a diastolic blood pressure of $<90 \mathrm{~mm} \mathrm{Hg}$ among those taking antihypertensive drugs. ${ }^{2,3}$ A full color version of this figure is available at the Hypertension Research journal online.

the morbidity rate from stroke was about four times higher than that from acute myocardial infarction. ${ }^{5,6,8}$ With respect to the subtype of stroke, recently, the morbidity rate from cerebral infarction was two to three times higher than that from cerebral hemorrhage. ${ }^{5,9}$ On the other hand, the morbidity rate from myocardial infarction has slightly increased in urban areas. ${ }^{6,10}$ In the Suita Study, the morbidity rate from stroke was about two times higher than that from acute myocardial infarction, showing a reduction in the difference. ${ }^{11,12}$
There is a graded, continuous, positive association between blood pressure level and risk for cardiovascular diseases. ${ }^{9,13-19}$ In a project to integrate major cohort studies in Japan, EPOCH-JAPAN, a meta-analysis of 10 cohort studies (total: $\sim 70,000$ persons) showed that the association between blood pressure level and cardiovascular disease mortality risk was almost logarithmically linear in middle-aged (40-64 years) and early-phase elderly (65-74 years) people. The slope was stronger in younger people, and the risk was lowest in those with optimal blood pressure (Figure 1-4). ${ }^{13}$ In late-phase elderly people 


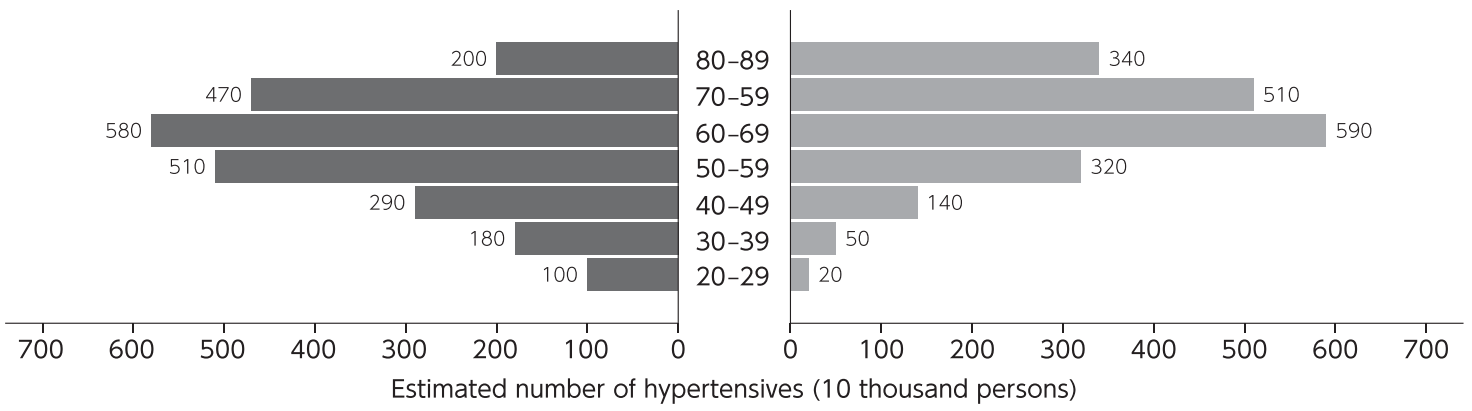

Figure 1-2 Estimated number of hypertensives in Japan (by sex/age group). Estimated from NIPPON DATA2010 and 2010 National Census of Japan. A full color version of this figure is available at the Hypertension Research journal online.
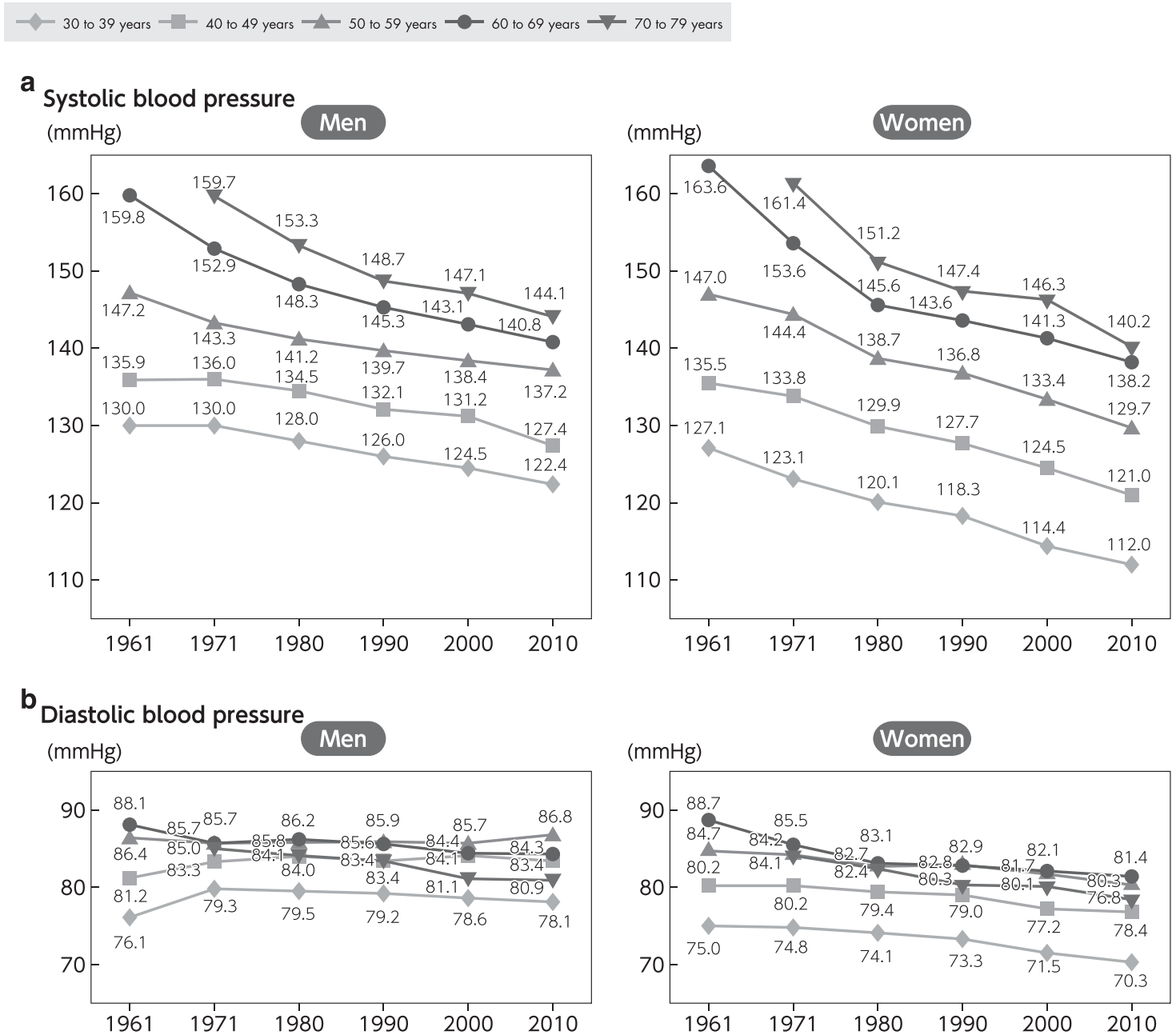

Figure 1-3 The 50-year trend in the average blood pressure (mm Hg) by sex and age group (1961-2010). 1st National Survey on Adult Diseases, 2nd National Survey on Adult Diseases, 3rd National Survey of Circulatory Disorders (NIPPON DATA80), 4th National Survey of Circulatory Disorders (NIPPON DATA90), 5th National Survey of Circulatory Disorders, NIPPON DATA2010. A full color version of this figure is available at the Hypertension Research journal online.

(75-89 years), the cardiovascular mortality risk also increased with blood pressure level. An analysis excluding deaths during the first 3 years of follow-up to eliminate the reverse causation indicated a significant increase in the risk from high-normal blood pressure. This association has been similarly observed when reviewing mortality due to all subtypes of stroke, cerebral infarction, cerebral hemorrhage or coronary heart disease. ${ }^{13,14}$ In particular, the association with mortality due to cerebral hemorrhage is stronger.

The results of cohort studies investigating morbidity as an outcome in Japan also showed a similar association. ${ }^{9,15,16}$ The associations 

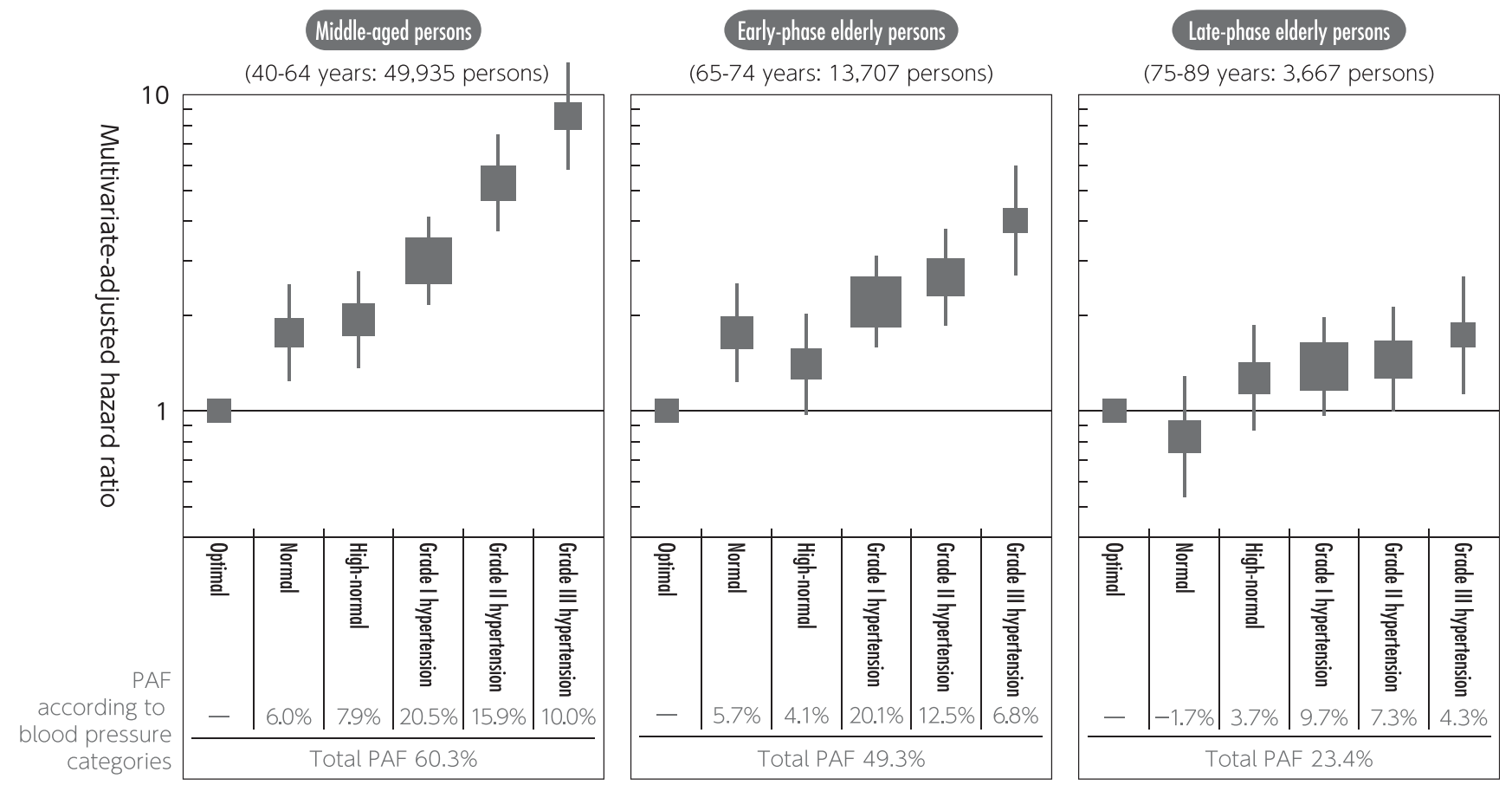

Figure 1-4 Hazard ratio and population-attributable fraction (PAF) for cardiovascular disease mortality according to blood pressure categories. EPOCHJAPAN: Meta-analysis of 10 cohorts in Japan (a total of 67000 men/women) by age group. Note 1: The hazard ratio was adjusted for age, sex, cohort, body mass index (BMI), serum total cholesterol, smoking and alcohol drinking. Note 2: PAF (population-attributable risk fraction) refers to the proportion of deceased cases in which death may have been prevented if all members of the population had shown optimal blood pressure. A full color version of this figure is available at the Hypertension Research journal online.

between blood pressure level and stroke/coronary heart disease morbidity risks were graded and continuous. The risks were the lowest in the optimal blood pressure group. The Hisayama Study clarified the association between lacunar infarction, as a subtype of cerebral infarction, and blood pressure. ${ }^{16}$

Recent cohort studies have indicated the population-ttributable fraction, which reflects the proportion of excess cardiovascular disease mortality/morbidity related to high blood pressure exceeding optimal values. ${ }^{9,13-16,20}$ According to EPOCH-JAPAN, high blood pressure exceeding optimal values explained $50 \%$ of all cardiovascular disease deaths, $52 \%$ of stroke deaths and $59 \%$ of deaths from coronary heart disease. The number of excess deaths from grade I hypertension was the largest. ${ }^{13}$ With respect to cardiovascular morbidity, this rate was also reported. In the Japan Public Health Center Study, excess stroke morbidity due to high blood pressure exceeding optimal values was $64 \%$ in men and $50 \%$ in women. In the Hisayama Study, the proportions were 52, 36, 76 and $25 \%$ for stroke, cerebral infarction, cerebral hemorrhage and coronary heart disease, respectively. ${ }^{15,20}$ The Circulatory Risk in Communities Study indicated that the portion of excess stroke morbidity from mild hypertension has been increasing and that from severe hypertension has been decreasing. ${ }^{9}$ Lifestyle modification in persons with high-normal blood pressure or grade I hypertension and strategies to prevent the development of hypertension are further important.

\section{2) Hypertension and other conditions/total mortality}

Hypertension increases the risk for chronic kidney disease and endstage renal disease. ${ }^{21-23} \mathrm{~A}$ cohort study in Okinawa showed that the future risk of end-stage renal disease increased by $\sim 30 \%$ per $10-\mathrm{mmHg}$ increase in systolic blood pressure. ${ }^{21}$ The Hisayama Study indicated that hypertension, especially in middle-aged persons,

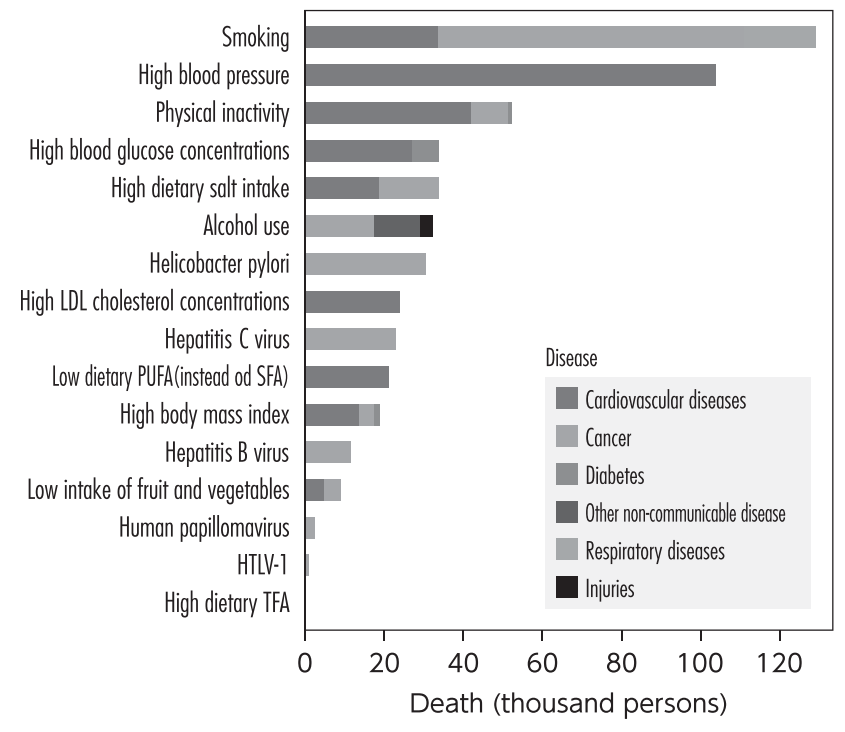

Figure 1-5 The number of deaths from noncommunicable diseases and injuries that were attributable to risk factors in Japan in 2007 (total of men and women). ${ }^{27} \mathrm{~A}$ full color version of this figure is available at the Hypertension Research journal online.

increased the risk for senile vascular dementia. ${ }^{24}$ Another study reported that middle-age hypertension increased the risk of reduction in future activities of daily living. ${ }^{25}$

Hypertension also increases the total mortality risk through various diseases described above. A meta-analysis involving 13 cohorts in Japan (total: 180000 persons; EPOCH-JAPAN) revealed that the total mortality risk elevated with an increase in blood pressure in both men 
and women aged 40-89 years. $^{26}$ It was estimated that high blood pressure exceeding optimal values could be attributed to $\sim 20 \%$ of allcause deaths. An estimation on the basis of the results of previous epidemiological studies showed that hypertension is the second most important factor of death in Japan, following smoking, and the annual number of deaths due to hypertension was estimated to be $\sim 100000$ (Figure 1-5). ${ }^{27}$ According to NIPPON DATA80, the average hypertension-related shortening of life expectancy was 2.2 and 2.9 years in men and women, respectively. ${ }^{28}$

\section{3) Accumulation of risk factors, metabolic syndrome and risk for cardiovascular disease}

When other established risk factors accumulate in the presence of hypertension, the risk for cardiovascular diseases increases further. ${ }^{29-33}$ Many cohort studies in Japan and their meta-analyses have revealed an increase in cardiovascular disease risk with the accumulation of smoking, diabetes, hypercholesterolemia or chronic kidney disease in the presence of hypertension.

Metabolic syndrome is also a condition involving high blood pressure as a factor. Many cohort studies in Japan have reported a metabolic syndrome-related increase in the risk for cardiovascular diseases. Cardiovascular disease morbidity and mortality risks increased 1.5- to 2.4-fold. ${ }^{34-36}$ On the other hand, several cohort studies investigating cardiovascular disease morbidity/mortality as an end point ${ }^{37-42}$ have suggested that the accumulation of metabolic risk factors is important regardless of the presence or absence of obesity. In addition, the integrated analysis of 10 cohort studies including these also confirmed this. ${ }^{43}$

\section{4) Various blood pressure parameters and cardiovascular disease risk}

With respect to the association between various blood pressure parameters and the risk for cardiovascular diseases, large-scale metaanalyses have shown that systolic blood pressure most strongly predicts the future risk. ${ }^{4-46}$ In a meta-analysis involving 16 cohorts in Japan (the Japan Arteriosclerosis Longitudinal Study), systolic blood pressure was the strongest predictor of stroke morbidity risk in both middle-aged/elderly men and women, followed by diastolic blood pressure. The prediction ability of pulse pressure was less strong. ${ }^{44}$ Concerning hemorrhagic stroke morbidity in men, diastolic blood pressure was the strongest predictor.

In most studies investigating the association between blood pressure and risk for cardiovascular diseases, blood pressure measured in the clinic or during health checkup was used. However, the risk-prediction ability of blood pressure measured at home has been reported to be stronger. ${ }^{47}$ Furthermore, ambulatory 24 -h blood pressure is also more useful than clinic blood pressure for predicting the cardiovascular disease risk. ${ }^{48-50}$

\section{CHARACTERISTICS OF HYPERTENSION IN THE JAPANESE}

\section{1) High salt intake}

An excessive intake of salt was one of the causes for the high prevalence of hypertension and stroke in the past in Japan. A high salt intake increases blood pressure. The INTERSALT Study showed that blood pressure was high in groups with a high salt intake estimated by $24-\mathrm{h}$ urine collection, and that a positive correlation was present between salt intake and blood pressure in individuals. $^{51}$

Although few studies have strictly evaluated salt intake using 24-h urine collection in the general population, the mean salt intake of men and women was 12.3 and $10.9 \mathrm{~g}$ per day, respectively, according to the
INTERMAP Study, in which salt intake was measured in men and women aged 40-59 years in Hokkaido, Toyama, Shiga and Wakayama between 1996 and $1999 .{ }^{52}$ The results of the National Health and Nutrition Survey in 2011 showed that the mean daily salt intake per person was $10.4 \mathrm{~g}$ (men: $11.4 \mathrm{~g}$, women: $9.4 \mathrm{~g}$ ); it has gradually decreased. ${ }^{53}$ According to previous studies using 24-h urine collection, salt intake in the Tohoku District in the 1950s was estimated to reach 25 g per day. ${ }^{54}$

In the Dietary Reference Intake in Japanese (2010), the dietary goal of salt intake to be achieved in adult men and women in the next 5 years is $<9.0$ and $<7.5 \mathrm{~g}$ per day, respectively. ${ }^{55}$ However, according to the National Health and Nutrition Survey in 2009, salt intake exceeded the target value in $\sim 70 \%$ of men and women. Health Japan 21 (II)(2012) targeted a reduction in the average salt intake of the Japanese to $8.0 \mathrm{~g}$ before $2022 .{ }^{56}$ In the World Health Organization guidelines on sodium intake, which were published in 2012, it is recommended that salt intake should be reduced to $<5 \mathrm{~g}$ per day in adults. ${ }^{57}$ The current status in Japan is still far from this recommendation.

The INTERSALT Study estimated that a decrease of $6 \mathrm{~g}$ per day in salt intake would reduce an increase in systolic pressure after 30 years of age by $10-11 \mathrm{~mm} \mathrm{Hg} .{ }^{51}$ Further efforts to reduce salt intake are necessary for preventing hypertension in Japan.

2) Increases in the prevalence of obesity and metabolic syndrome Obesity is less common in Japan than in other developed, industrialized countries. However, body mass index $\left(\mathrm{kg} \mathrm{m}^{-2}\right)$, which is an index of obesity, is found to increase substantially in men; the proportion of obesity in men has increased by about twofold during the past 30 years. ${ }^{58}$ According to the National Health and Nutrition Survey in 2011, the proportion of obesity (body mass index: $25 \mathrm{~kg} \mathrm{~m}^{-2}$ or more) in men aged 20-69 years was $32 \% .{ }^{53}$ On the other hand, there was no overall increase in the proportion of obesity in women.

Regarding the characteristics of hypertensive Japanese, lean hypertensives with a high salt intake account for a high percentage, but the number of obese hypertensives has recently increased, particularly among men. According to NIPPON DATA, the proportion of hypertensive men in whom obesity contributed to hypertension increased from 11 to $27 \%$ between 1980 and 2010, and that of hypertensive women from 19 to $26 \% .^{2}$ This would reflect an increase in the prevalence of metabolic syndrome in Japan. The National Health and Nutrition Survey in 2010 showed that metabolic syndrome was strongly suspected in more than $30 \%$ of men aged over 50 years and in $\sim 20 \%$ of women aged over 60 years. ${ }^{1}$

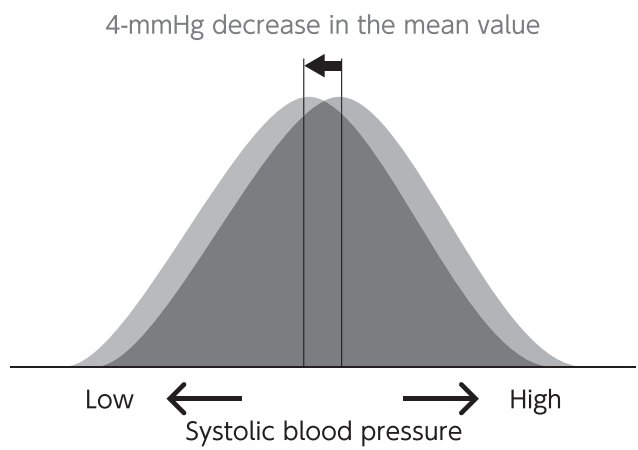

Figure 1-6 Population strategy to shift the distribution of systolic blood pressure in the Japanese to a lower level. A full color version of this figure is available at the Hypertension Research journal online. 


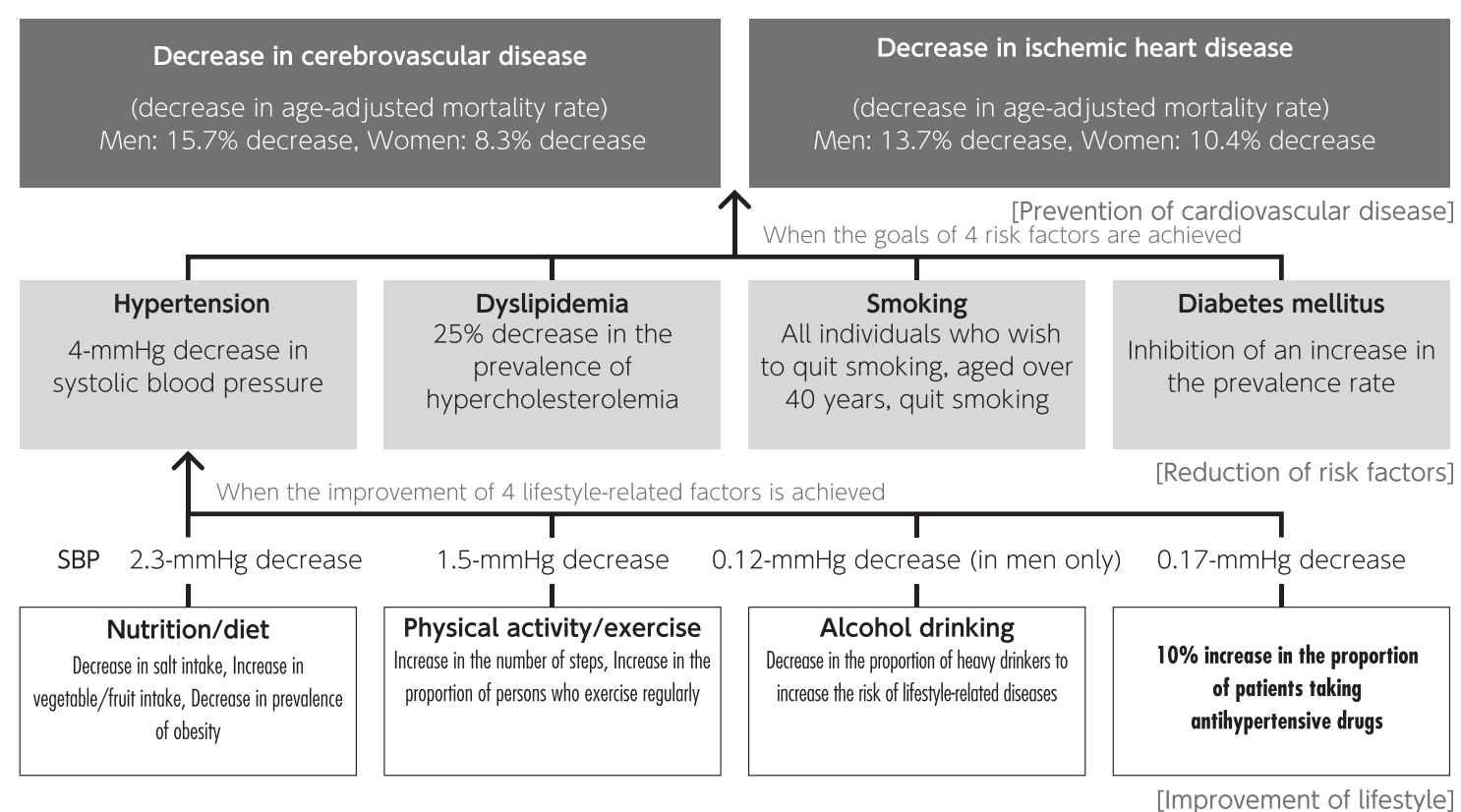

Figure 1-7 Target establishment for cardiovascular diseases in Health Japan 21 (II). ${ }^{62}$ A full color version of this figure is available at the Hypertension Research journal online.

In the United States, the prevalence of obesity has markedly increased since 1990, and those with a body mass index of $30 \mathrm{~kg} \mathrm{~m}^{-2}$ or more account for more than $30 \%$ of the population. ${ }^{59}$ In Japan, the percentage is $\sim 3 \%$. However, it may increase with the westernization of lifestyle in the future. Strategies to prevent obesity must be promoted.

\section{PUBLIC HEALTH MEASURES AGAINST HYPERTENSION}

As shown by many epidemiological studies, more than half of high blood pressure-related excessive cardiovascular mortality/morbidity events occurred in people with mildly high blood pressure (grade I hypertension or lower). ${ }^{9,13-16}$ To reduce excess cardiovascular disease mortality/morbidity, high-risk strategies involving hypertensive patients alone are insufficient. Population strategies to lower the blood pressure distribution of the whole population (the whole Japanese nation) are necessary. ${ }^{60,61}$

The 'National Health-Promotion Project in the 21st Century' (Health Japan 21 (II)), which was announced by the Minister of Health, Labour and Welfare in 2012, targets a decrease in average systolic blood pressure in Japan by $4 \mathrm{~mm} \mathrm{Hg}$ (men: $138 \rightarrow 134 \mathrm{~mm} \mathrm{Hg}$, women: $133 \rightarrow 129 \mathrm{~mm} \mathrm{Hg}$ ) within 10 years (before 2022). ${ }^{56}$ The goal is to lower the blood pressure distribution of the whole Japanese nation (Figure 1-6).

The establishment of target values for cardiovascular diseases in Health Japan 21 (II) is presented in Figure 1-7. A 2.3-mm Hg decrease in systolic blood pressure is targeted through nutritional/dietary strategies such as reducing salt intake (to $8 \mathrm{~g}$ per day), increasing vegetable/fruit intake (to $350 \mathrm{~g}$ per day) and decreasing the number of obese people. A $1.5-\mathrm{mm} \mathrm{Hg}$ decrease is targeted through physical activity/exercise strategies $(\sim 1,500$ step increase in the number of steps). A $0.12-\mathrm{mm} \mathrm{Hg}$ decrease is targeted through alcohol strategies (decreasing the number of heavy drinkers). A $0.17-\mathrm{mm} \mathrm{Hg}$ decrease is targeted through strategies regarding antihypertensive therapy (increasing the compliance rate by $10 \%$ ). Overall, a $4-\mathrm{mm} \mathrm{Hg}$ decrease in systolic blood pressure is targeted. ${ }^{62}$
In Health Japan 21 (II), the cardiovascular disease-reducing effects of target achievement were estimated using the EPOCH-JAPAN database. ${ }^{62}$ Overall, the project targets to decrease age-adjusted mortality for stroke (cerebrovascular disease) in men and women by 15.7 and $8.3 \%$, respectively, and that for coronary heart disease (ischemic heart disease) by 13.7 and $10.4 \%$, respectively, as shown in Figure 1-7. To achieve these goals, the role of blood pressure reduction is important. In brief, only a $4-\mathrm{mm} \mathrm{Hg}$ decrease in average systolic blood pressure in the Japanese is estimated to reduce ageadjusted mortality from stroke in men and women by 8.9 and $5.8 \%$, respectively (the total number of deaths from stroke will decrease by $\sim 10000$ per year), and that for coronary heart disease by 5.4 and $7.2 \%$, respectively (the total number of deaths from coronary heart disease will decrease by $\sim 5000$ per year).

Population strategies to achieve the above targets include environmental approaches from various aspects, such as mass media-mediated public education, obligations regarding the labeling of salt content by food manufacturers and menu improvement/promotion of nutrition labeling in school lunch/food-service industries. ${ }^{63,64}$ It is necessary to promote high-risk strategies in parallel with population strategies. Screening and health guidance are important. ${ }^{65}$ All health/medical specialists, including physicians, nurses, public health nurses, school nurses, dietitians and pharmacists, must instruct all persons including high-risk individuals to improve their diet (salt reduction/maintenance of optimal body weight), increase physical activities and maintain moderate alcohol consumption in health care/medical practice.

\section{Citation Information}

We recommend that any citations to information in the Guidelines are presented in the following format:

The Japanese Society of Hypertension Guidelines for the Management of Hypertension (JSH 2014). Hypertens Res 2014; 37: 253-392.

Please refer to the title page for the full list of authors. 